\title{
Serum tenascin- $C$ is independently associated with increased major adverse cardiovascular events and death in individuals with type 2 diabetes: a French prospective cohort
}

\author{
Barnabas Gellen $^{1}$ (D) N Nathalie Thorin-Trescases ${ }^{2}$ • Eric Thorin ${ }^{2,3}$ • Elise Gand ${ }^{4}$ - Philippe Sosner ${ }^{4,5,6} \cdot$ Sonia Brishoual $^{4}$. \\ Vincent Rigalleau $^{7} \cdot$ David Montaigne ${ }^{8,9} \cdot$ Vincent Javaugue $^{4,10} \cdot$ Yann Pucheu $^{11} \cdot$ Philippe Gatault $^{12} \cdot$ Xavier Piguel $^{13}$. \\ Samy Hadjadj ${ }^{12,14}$. Pierre-Jean Saulnier ${ }^{4}$. on behalf of the SURDIAGENE Study group
}

Received: 20 November 2019 / Accepted: 13 January 2020 / Published online: 10 February 2020

(C) Springer-Verlag GmbH Germany, part of Springer Nature 2020

\begin{abstract}
Aims/hypothesis Tenascin-C (TN-C) is an extracellular matrix glycoprotein highly expressed in inflammatory and cardiovascular (CV) diseases. Serum TN-C has not yet been specifically studied in individuals with type 2 diabetes, a condition associated with chronic low-grade inflammation and increased CV disease risk. In this study, we hypothesised that elevated serum TN-C at enrolment in participants with type 2 diabetes would be associated with increased risk of death and major adverse CV events (MACE) during follow-up.

Methods We used a prospective, monocentric cohort of consecutive type 2 diabetes participants (the SURDIAGENE [SUivi Rénal, DIAbète de type 2 et GENEtique] cohort) with all-cause death as a primary endpoint and MACE (CV death, non-fatal myocardial infarction or stroke) as a secondary endpoint. We used a proportional hazard model after adjustment for traditional risk factors and the relative integrated discrimination improvement (rIDI) to assess the incremental predictive value of TN-C for these risk factors. Results We monitored 1321 individuals (58\% men, mean age $64 \pm 11$ years) for a median of 89 months. During follow-up, 442 individuals died and 497 had MACE. Multivariate Cox analysis showed that serum TN-C concentrations were associated with an increased risk of death (HR per 1 SD: 1.27 [95\% CI 1.17, 1.38]; $p<0.0001$ ) and MACE (HR per 1 SD: 1.23 [95\% CI 1.13, 1.34]; $p<0.0001$ ). Using TN-C concentrations on top of traditional risk factors, prediction of the risk of all-cause death (rIDI: $8.2 \%$; $p=$ 0.0006 ) and MACE (rIDI: $6.7 \% ; p=0.0014$ ) improved significantly, but modestly.

Conclusions/interpretation In individuals with type 2 diabetes, increased serum TN-C concentrations were independently associated with death and MACE. Therefore, including TN-C as a prognostic biomarker could improve risk stratification in these individuals.
\end{abstract}

Electronic supplementary material The online version of this article (https://doi.org/10.1007/s00125-020-05108-5) contains peer-reviewed but unedited supplementary material, which is available to authorised users.

Barnabas Gellen

barnabas.gellen.cardio@gmail.com

1 ELSAN, Polyclinique de Poitiers, 1 Rue de la Providence, F-86000 Poitiers, France

2 Research Center, Montreal Heart Institute, Montreal, QC, Canada

3 Department of Surgery, Faculty of Medicine, Montreal Heart Institute, Université de Montréal, Montreal, QC, Canada

4 INSERM, Centre d'Investigation Clinique CIC1402, Université de Poitiers, CHU de Poitiers, Poitiers, France

5 Laboratoire MOVE (EA 6314), Université de Poitiers, Poitiers, France

6 Centre Médico-Sportif Mon Stade, Paris, France
Endocrinology - Diabetology - Nutrition, CHU de Bordeaux, Hôpital Haut-Lévêque, Pessac, France

8 Department of Clinical Physiology - Echocardiography, CHU Lille, Lille, France

9 INSERM U1011, EGID, Institut Pasteur de Lille, University of Lille, Lille, France

10 Nephrology, CHU de Poitiers, Poitiers, France

11 Cardiology, CHU de Bordeaux, Pessac, France

12 Transplantation, Immunology and Inflammation (T2I) - EA4245, CHRU de Tours, Nephrology-Hypertension, Dialysis and Renal Transplantation, FHU SUPORT, Université de Tours, Tours, France

13 Endocrinology—Diabetology, CHU de Poitiers, Poitiers, France

14 L'Institut du Thorax, INSERM, CNRS, UNIV Nantes, CHU Nantes, Nantes, France 


\section{Research in context}

What is already known about this subject?

- Tenascin-C (TN-C) is an extracellular matrix circulating glycoprotein that is highly expressed in inflammatory and cardiovascular (CV) diseases. It is, thus, an emerging candidate biomarker for diagnosis and/or risk stratification in individuals with various CV diseases

- Serum TN-C has not yet been specifically measured in individuals with type 2 diabetes, a condition associated with chronic low-grade inflammation and increased CV risk

What is the key question?

- Are elevated serum TN-C levels at baseline (study enrolment) in individuals with type 2 diabetes associated with risk of death and major adverse CV events (MACE) during follow-up?

What are the new findings?

- In individuals with type 2 diabetes ( $n=1321)$ that were followed for 89 months, serum TN-C concentrations were independently associated with an increased risk of all-cause death (HR per 1 SD: 1.27 [95\% CI 1.17, 1.38]; $p<0.0001$ ) and MACE (HR per 1 SD: 1.23 [95\% CI 1.13, 1.34]; $p<0.0001)$

- In addition, using TN-C concentrations on top of traditional risk factors, prediction of the risk of all-cause death (rIDI: $8.2 \% ; p=0.0006$ ) and MACE (rIDI: $6.7 \% ; p=0.0014$ ) improved modestly, but significantly

How might this impact on clinical practice in the foreseeable future?

- Including TN-C as a prognostic biomarker could improve risk stratification in individuals with type 2 diabetes

Keywords Cardiovascular risk $\cdot$ MACE $\cdot$ Tenascin-C $\cdot$ Type 2 diabetes

$\begin{array}{ll}\text { Abbreviations } & \\ \text { ANGPTL2 } & \text { Angiopoietin-like 2 } \\ \text { CAD } & \text { Coronary artery disease } \\ \text { CKD } & \text { Chronic kidney disease } \\ \text { CRP } & \text { C-reactive protein } \\ \text { CV } & \text { Cardiovascular } \\ \text { ECM } & \text { Extracellular matrix } \\ \text { LV } & \text { Left ventricular } \\ \text { MACE } & \text { Major adverse cardiovascular events } \\ \text { MI } & \text { Myocardial infarction } \\ \text { NT-proBNP } & \text { N-terminal pro-B-type natriuretic peptide } \\ \text { rIDI } & \text { Relative integrated discrimination } \\ & \text { improvement } \\ \text { SBP } & \text { Systolic BP } \\ \text { SURDIAGENE } & \text { SUivi Rénal, DIAbète de type 2 et } \\ & \text { GENEtique } \\ \text { TN-C } & \text { Tenascin-C } \\ \text { TNFR1 } & \text { TNF receptor 1 }\end{array}$

\section{Introduction}

The major causes of morbidity and mortality in individuals with type 2 diabetes are cardiovascular (CV) complications [1]. Type 2 diabetes is associated with a twofold increased risk of death and a threefold increased risk of coronary artery disease (CAD) [2]. CV disease risk stratification in those with type 2 diabetes is typically based on the evaluation of classical $\mathrm{CV}$ risk factors and on diabetes-related variables [3, 4]. Moreover, single biomarkers associated with atherosclerosis (e.g. osteopontin and galactin-3 [5, 6]), inflammation (e.g. TNF receptor 1 [TNFR1] and angiopoietin-like $2[7,8])$ and congestive heart failure (e.g. N-terminal pro-B-type natriuretic peptide [NT-proBNP] $[9,10])$, or a combination of biomarkers [11-13] have been proposed to improve risk stratification in individuals with type 2 diabetes. Although promising data have been obtained using these biomarkers, up until now, none of them have been incorporated into international guidelines [1]. Thus, the research for new predictive biomarkers of $\mathrm{CV}$ disease in type 2 diabetes remains highly relevant.

Tenascin-C (TN-C) is a large hexameric extracellular matrix (ECM) glycoprotein expressed in most normal adult tissues $[14,15]$. It is implicated in the regulation of cell behaviour and tissue remodelling in many developmental and pathological processes $[14,16]$. At sites of tissue injury and inflammation, TN-C is markedly upregulated $[17,18]$ : it is transiently upregulated during acute inflammation, such as after acute myocardial infarction (MI), during which serum TN-C levels rise within hours and peak after 5 days, and may be used to predict long-term CV events $[19,20]$. In 
chronic inflammation and during tissue repair, TN-C levels are continuously upregulated [16]. Beyond acute coronary events, TN-C is an emerging candidate biomarker for diagnosis and/or risk stratification in various $\mathrm{CV}$ diseases, such as aortic aneurysm and dissection [21], dilated cardiomyopathy [22] and atherosclerosis [23]. TN-C is consequently considered as a marker of inflammation in $\mathrm{CV}$ diseases.

In individuals with diabetes, a significant association between increased TN-C levels and the severity of proliferative diabetic retinopathy has been reported [24-26]. There are no clear data in the literature examining the prognostic usefulness of serum TN-C levels for CV outcomes specifically involving type 2 diabetes. Recently, a big-data approach in $>8400$ individuals with dysglycaemia identified TN-C, among other biomarkers, as an independent determinant of $\mathrm{CV}$ outcomes or death $[11,12]$. In individuals with chronic kidney disease (CKD; $40 \%$ with type 2 diabetes), higher serum TN$\mathrm{C}$ levels were significantly associated with $\mathrm{CV}$-associated morbidity and/or death [27, 28]. The association between $\mathrm{TN}-\mathrm{C}$ and $\mathrm{CV}$ events in a population of purely diabetic individuals remains to be demonstrated.

In the present study, we examined the association of TN-C with all-cause death and CV-associated morbidity in a prospective cohort of individuals with type 2 diabetes [7, 8 , 29]. We also assessed the incremental prognostic value of serum TN-C concentration in addition to established CV risk markers for long-term risk assessment of individuals with type 2 diabetes.

\section{Methods}

Participants from the SURDIAGENE (SUivi Rénal, DIAbète de type 2 et GENEtique) cohort were recruited at the University Hospital of Poitiers, France, from 2002 to 2012 [29]. The local ethics committee (CPP Ouest III) approved the study design and written informed consent was obtained from all participants. Participants were prospectively followed-up until death, or until 31 December 2015, whichever came first.

Participants with a baseline eGFR $<30 \mathrm{ml} \mathrm{min}^{-1}$ $[1.73 \mathrm{~m}]^{-2}$ and/or a history of prior renal replacement therapy and/or a follow-up duration of $<3$ months were excluded from the present analysis. Demographic and clinical data, morphometric measurements, and ECG and biological variables were obtained at baseline. A self-reported history of MI or symptomatic peripheral artery disease was noted.

Assays Blood samples and second morning urine samples were obtained in individuals after an overnight fast. $\mathrm{HbA}_{1 \mathrm{c}}$ and serum creatinine concentrations were centrally determined using a chromatography method (ADAMS A1c HA8160 analyser; Menarini, Florence, Italy) and a colorimetric method on an automated analyser (KONE Optima; Thermo Clinical Labsystems, Vantaa, Finland), respectively. eGFR was calculated using the Chronic Kidney Disease Epidemiology Collaboration formula [30]. Urinary creatinine was measured on a Hitachi 911 automatic analyser (Roche Diagnostics, Meylan, France), and urinary albumin by nephelometry on a Modular System P (Roche Diagnostics).

Samples were processed under standardised conditions and stored at $-80^{\circ} \mathrm{C}$ in the Poitiers Biological Resource Center (BRC BB-0033-00068, Poitiers, France), undergoing only one freeze-thaw cycle prior to assay. Plasma C-reactive protein (CRP) was measured at baseline using an immunoturbidimetric assay (Roche/Hitachi cobas c systems; Roche Diagnostics). Serum concentrations of TNF receptor 1 (soluble TNFR1) and angiopoietin-like 2 (ANGPTL2), two additional markers of inflammation, were measured using a human soluble TNFR1 ELISA kit (EKF Diagnostics, Dublin, Ireland) and a human ANGPTL2 ELISA kit (Cloud-Clone, Houston, TX, USA), according to the manufacturer's instructions $[7,8]$. Serum TN-C concentrations were measured using a commercially available enzyme immunoassay ELISA kit for large TN-C (catalogue no. JP27751; IBL International, Hamburg, Germany), according to the manufacturer's instructions. The limit of detection was $0.38 \mathrm{ng} / \mathrm{ml}$, the intra-assay coefficient of variability was $3.8-6.6 \%$, and the inter-assay CV was 3.9-7.0\%. Serum was diluted 1:8 in all samples.

Study outcomes The primary endpoint was all-cause mortality and the secondary endpoint was a composite of $\mathrm{CV}$ death, non-fatal $\mathrm{MI}$ and non-fatal stroke (major adverse $\mathrm{CV}$ events [MACE]) [31]. Vital status and CV endpoints were determined from participants' hospital records, interviews with their general practitioners and inquiry to the French National Death Registry. Specific cause of each death event was identified by the ICD code on the death certificate, which was issued by authorised clinical practitioners (ICD-9-CM before 1 January 2012, www.icd9data.com/2007/Volume1; ICD-10CM thereafter, http://apps.who.int/classifications/icd10/ browse/2016/en). The present analysis takes the most recent available data (December 2015) into account. Each endpoint was reviewed by an independent adjudication committee according to the international definitions of clinical outcomes. Deaths were categorised as being due to $\mathrm{CV}$ disease, malignant tumour, infection, renal causes and other causes. The adjudication committee was blinded with regard to TN-C concentrations. We defined history of $\mathrm{CV}$ disease by a history of $\mathrm{MI}$ and/or stroke.

Statistical analysis Qualitative variables are reported as absolute values and percentages, while quantitative variables are described by mean \pm SD or median (interquartile range; 25 th to 75 th percentile), as appropriate. Qualitative variables were compared between tertile groups using the $\chi^{2}$ test, whereas 
Table 1 Baseline participant characteristics according to tertiles of serum TN-C concentration

\begin{tabular}{|c|c|c|c|c|c|}
\hline Characteristic & All $(n=1321)$ & Tertile $1(n=440)$ & Tertile $2(n=440)$ & Tertile $3(n=441)$ & $p$ value \\
\hline TN-C, ng/ml (range) & & $8.84-50.96$ & $50.97-81.55$ & $81.56-317.58$ & \\
\hline Men, $n(\%)$ & $762(58)$ & $241(55)$ & $250(57)$ & $271(61)$ & 0.1208 \\
\hline Age, years & $64 \pm 11$ & $62 \pm 10$ & $64 \pm 11$ & $67 \pm 11$ & $<0.0001$ \\
\hline Non-white ethnicity, $n(\%)$ & $43(3)$ & $16(4)$ & $19(4)$ & $8(2)$ & 0.0958 \\
\hline BMI, $\mathrm{kg} / \mathrm{m}^{2}$ & $31 \pm 6$ & $32 \pm 6$ & $32 \pm 6$ & $31 \pm 6$ & 0.0405 \\
\hline SBP, $\mathrm{mmHg}^{\mathrm{a}}$ & $132 \pm 17$ & $131 \pm 16$ & $132 \pm 17$ & $133 \pm 18$ & 0.1090 \\
\hline $\mathrm{DBP}, \mathrm{mmHg}^{\mathrm{a}}$ & $73 \pm 11$ & $72 \pm 11$ & $73 \pm 11$ & $73 \pm 11$ & 0.7471 \\
\hline Resting HR, bpm ${ }^{\mathrm{a}}$ & $71 \pm 14$ & $70 \pm 13$ & $71 \pm 14$ & $72 \pm 14$ & 0.0182 \\
\hline Sinus rhythm, $n(\%)^{\mathrm{a}}$ & $1251(95)$ & $424(97)$ & $423(96)$ & $404(92)$ & 0.0009 \\
\hline Active smoking, $n(\%)$ & $148(11)$ & $55(13)$ & $46(10)$ & $47(11)$ & 0.5702 \\
\hline Hypertension, $n(\%)$ & $1090(83)$ & $366(83)$ & $358(81)$ & $366(83)$ & 0.7372 \\
\hline History of CV disease, $n(\%)$ & 253 (19) & $70(16)$ & $78(18)$ & $105(24)$ & 0.0077 \\
\hline Retinopathy, $n(\%)^{\mathrm{a}}$ & & & & & 0.0351 \\
\hline None & $754(58)$ & $260(59)$ & $248(57)$ & $246(57)$ & \\
\hline Background & $383(29)$ & $131(30)$ & $139(32)$ & $113(26)$ & \\
\hline Severe & $87(7)$ & $21(5)$ & $24(6)$ & $42(10)$ & \\
\hline Proliferative & $81(6)$ & $25(6)$ & $24(6)$ & $32(7)$ & \\
\hline Nephropathy, $n(\%)^{\mathrm{ab}}$ & & & & & 0.4276 \\
\hline Normoalbuminuria & $534(45)$ & $183(48)$ & $173(44)$ & $178(44)$ & \\
\hline Microalbuminuria & $423(36)$ & $134(35)$ & $150(38)$ & $139(35)$ & \\
\hline Macroalbuminuria & $222(19)$ & $63(17)$ & $74(19)$ & $85(21)$ & \\
\hline Diabetes duration, years ${ }^{\mathrm{a}}$ & $12(6-20)$ & $12(5-18)$ & $12(6-20)$ & $14(7-22)$ & 0.0017 \\
\hline $\mathrm{HbA}_{1 \mathrm{c}}, \%^{\mathrm{a}}$ & $7.82 \pm 1.56$ & $7.86 \pm 1.62$ & $7.73 \pm 1.43$ & $7.87 \pm 1.62$ & 0.6831 \\
\hline $\mathrm{HbA}_{1 \mathrm{c}}, \mathrm{mmol} / \mathrm{mol}^{\mathrm{a}}$ & $62 \pm 13$ & $62 \pm 13$ & $61 \pm 11$ & $63 \pm 13$ & \\
\hline LDL-cholesterol, $\mathrm{mmol} / \mathrm{l}^{\mathrm{a}}$ & $2.72 \pm 0.95$ & $2.68 \pm 0.99$ & $2.72 \pm 0.93$ & $2.77 \pm 0.95$ & 0.4071 \\
\hline Serum creatinine, $\mu \mathrm{mol} / 1$ & $81(68-97)$ & $79(65-93)$ & $80(68-96)$ & $84(71-102)$ & $<0.0001$ \\
\hline 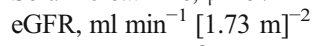 & $76.6 \pm 20.9$ & $80.2 \pm 20.5$ & $77.1 \pm 20.3$ & $72.5 \pm 21.3$ & $<0.0001$ \\
\hline $\mathrm{uACR}, \mathrm{mg} / \mathrm{mmol}^{\mathrm{a}}$ & $2.7(1.0-10.7)$ & $2.1(0.9-10.1)$ & $2.8(1.0-9.1)$ & $3.4(1.1-14.4)$ & 0.0064 \\
\hline $\mathrm{CRP}, \mathrm{mg} / \mathrm{l}^{\mathrm{a}}$ & $3.2(1.4-7.1)$ & $2.5(1.2-5.1)$ & $3.2(1.3-7.3)$ & $3.7(1.9-9.8)$ & $<0.0001$ \\
\hline ANGPTL2, ng/ml & $15(11-19)$ & $14(11-19)$ & $14(11-19)$ & $16(12-22)$ & $<0.0001$ \\
\hline NT-proBNP, pg/ml ${ }^{\mathrm{a}}$ & $103(48-262)$ & $71(36-162)$ & $96(47-243)$ & $178(64-472)$ & $<0.0001$ \\
\hline sTNFR1, pg/ml ${ }^{\mathrm{a}}$ & $1822(1548-2243)$ & $1700(1473-2068)$ & $1789(1550-2250)$ & $1960(1633-2568)$ & $<0.0001$ \\
\hline \multicolumn{6}{|l|}{ Medication, $n(\%)$} \\
\hline Beta blockers & 447 (34) & $154(35)$ & 149 (34) & $144(33)$ & 0.7626 \\
\hline ACE inhibitor & 493 (37) & $162(37)$ & $159(36)$ & $172(39)$ & 0.6556 \\
\hline $\mathrm{ARB}$ & $370(28)$ & $125(28)$ & $134(30)$ & $111(25)$ & 0.2120 \\
\hline Diuretics & $588(45)$ & $201(46)$ & $188(43)$ & $199(45)$ & 0.6446 \\
\hline Insulin & $781(59)$ & $248(56)$ & $256(58)$ & $277(63)$ & 0.1333 \\
\hline Statin & $606(46)$ & $207(47)$ & $201(46)$ & $198(45)$ & 0.8110 \\
\hline
\end{tabular}

Quantitative variables are described as mean $\pm \mathrm{SD}$ or median (25th-75th percentile), unless otherwise specified $p$ values are for comparison between tertiles by $\chi^{2}$ test

${ }^{\text {a }}$ Missing values: SBP, $n=7$ (tertile 1, $n=3$; tertile 3, $n=4$ ); DBP, $n=7$ (tertile 1, $n=3$; tertile 3, $n=4$ ); resting HR, $n=6$ (tertile 1,n=3; tertile 2, $n=1$; tertile 3,n=2); sinus rhythm, $n=6$ (tertile $1, n=3$; tertile 2, $n=1$; tertile 3, $n=2$ ); retinopathy, $n=16$ (tertile $1, n=3$; tertile 2,n=5; tertile 3,n=8); nephropathy, $n=142$ (tertile $1, n=60$; tertile 2, $n=; 43$ tertile $3, n=39$ ); diabetes duration, $n=2$ (tertile $1, n=1 ;$ tertile $3, n=1) ; \mathrm{HbA}_{1 \mathrm{c}}, n=1$ (tertile $2 n=$ 1); LDL-cholesterol, $n=51$ (tertile 1, $n=27$; tertile 2, $n=10$; tertile 3, $n=14$ ); uACR, $n=4$ (tertile 2, $n=1$; tertile 3, $n=3$ ); CRP, $n=65$ (tertile 1, $n=34$; tertile 2, $n=15$; tertile 3, $n=16$ ); NT-proBNP, $n=1$ (tertile 2, $n=1)$; sTNFR1, $n=1$ (tertile $1, n=1$ )

${ }^{\mathrm{b}}$ Nephropathy status was defined as follows: normoalbuminuria, uACR $<30 \mathrm{mg} / \mathrm{g}$ microalbuminuria; uACR $30-300 \mathrm{mg} / \mathrm{g}$; macroalbuminuria, uACR $>300 \mathrm{mg} / \mathrm{g}$

ACE, angiotensin-converting-enzyme; ARB, angiotensin II receptor blocker; DBP, diastolic BP; HR, heart rate; sTNFR1, soluble TNFR1; uACR, urinary albumin/creatinine ratio

quantitative variables were compared using the Student's $t$ test or Mann-Whitney $U$ test, as appropriate. Correlation between quantitative variables was tested using a Spearman's test. The time to event was plotted by the Kaplan-Meier method curves according to tertiles of TN-C, and compared with the logrank test. Risk-prediction models established by Cox proportional hazard models were used to analyse the effect on study outcomes. The HR and 95\% CI are presented.
We tested each model for log-linearity and proportionality assumptions and covariates were log-transformed if appropriate.

We adjusted the risk of all-cause death and MACE for predetermined covariates (age, sex, active smoking, statin treatment, hypertension, insulin treatment, eGFR and history of $\mathrm{CV}$ disease) as they represent established key risk factors for our outcomes. The relative integrated discrimination 
Fig. 1 Kaplan-Meier survival curves according to TN-C tertiles for (a) overall risk of death and (b) MACE. $p$ values were calculated using the logrank test
Tertile 1 - Tertile $2-$ Tertile 3

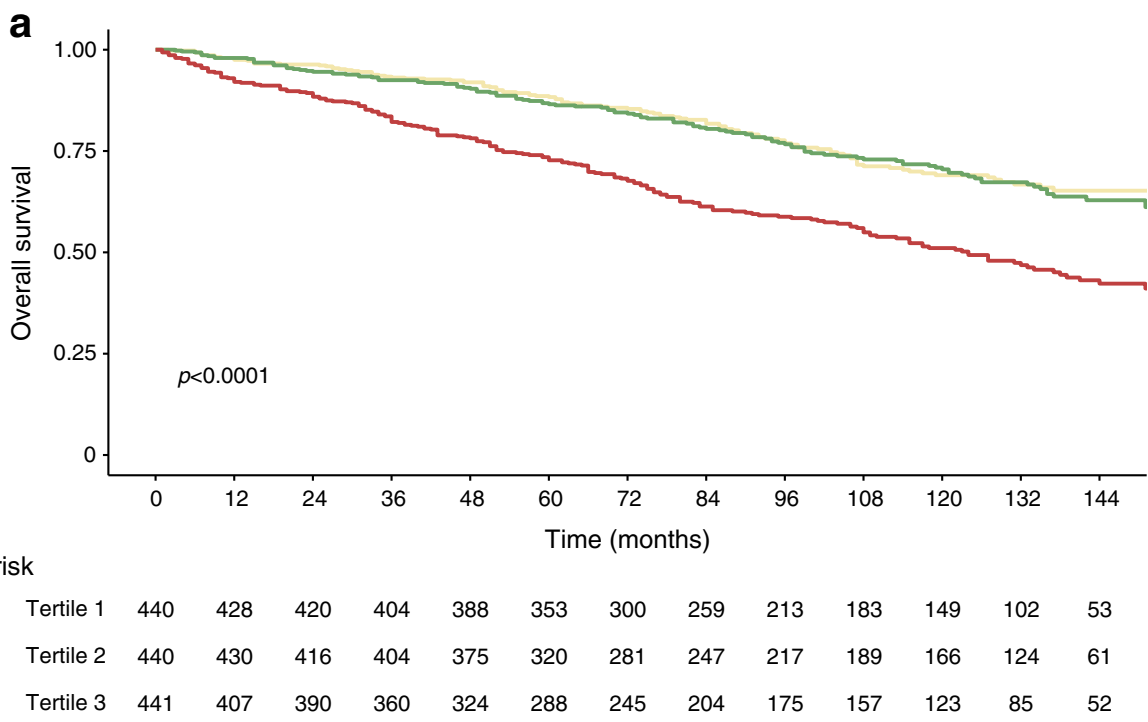

No. at risk

$\begin{array}{llllllllllllll}\text { Tertile 1 } & 440 & 428 & 420 & 404 & 388 & 353 & 300 & 259 & 213 & 183 & 149 & 102 & 53 \\ \text { Tertile 2 } & 440 & 430 & 416 & 404 & 375 & 320 & 281 & 247 & 217 & 189 & 166 & 124 & 61 \\ \text { Tertile 3 } & 441 & 407 & 390 & 360 & 324 & 288 & 245 & 204 & 175 & 157 & 123 & 85 & 52\end{array}$

b

- Tertile 1 - Tertile 2 - Tertile 3

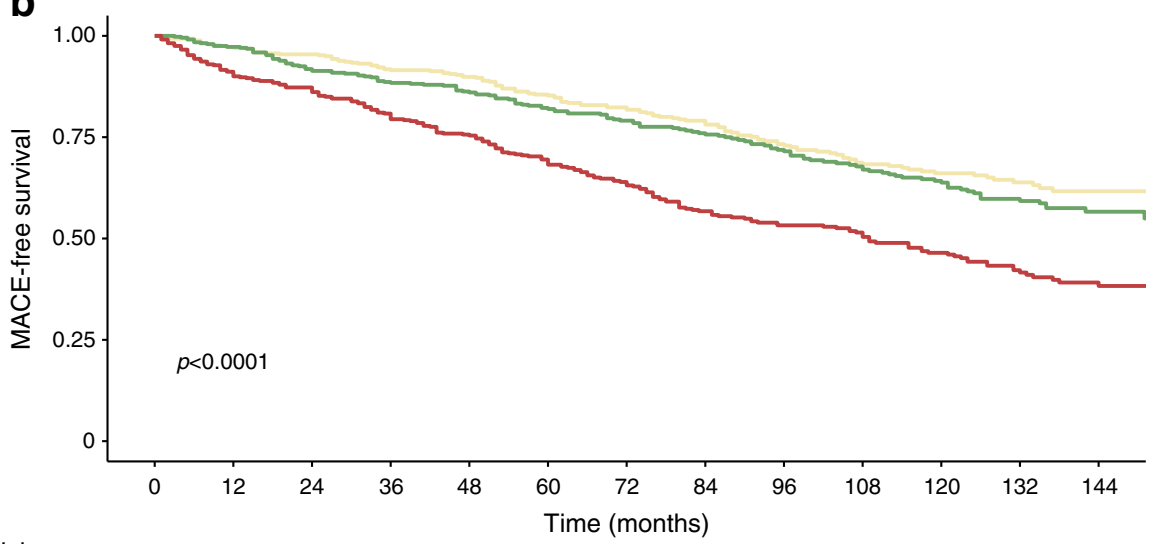

No. at risk

$\begin{array}{llllllllllllll}\text { Tertile 1 } & 440 & 425 & 416 & 397 & 379 & 340 & 286 & 246 & 198 & 174 & 141 & 98 & 51 \\ \text { Tertile 2 } & 440 & 427 & 403 & 388 & 358 & 303 & 262 & 233 & 202 & 174 & 152 & 112 & 57 \\ \text { Tertile 3 } & 441 & 399 & 381 & 348 & 312 & 271 & 228 & 188 & 158 & 143 & 109 & 73 & 46\end{array}$

improvement (rIDI) index was calculated to assess the improvement in 5 year risk prediction of $\mathrm{TN}-\mathrm{C}$ in addition to these predetermined risk factors. The 5 year risk was selected since it represents clinically relevant information. Harrell's $\mathrm{C}$-index was also determined to assess discrimination of the multivariate models. Improvement of increment of TN-C was assessed by bootstrap procedure.

We performed three sensitivity analyses to account for biomarkers of inflammation (CRP, ANGPTL2 and TNFR1), a marker of heart failure (NT-proBNP), additional CV risk factors, such as BMI, $\mathrm{HbA}_{1 \mathrm{c}}$, diabetes duration, LDL levels, systolic BP (SBP), and the presence of diabetic retinopathy.

All hypotheses were tested at the $5 \%$ level of significance. Statistical analyses were carried out using the SAS version 9.4 software package (SAS, Cary, NC, USA).

\section{Results}

Out of 1468 participants, the 1321 who met the selection criteria, had available serum samples and had complete covariate data were analysed. The demographic, clinical and biological characteristics of the study population are summarised in Table 1, according to TN-C tertiles.

Higher TN-C concentrations were significantly associated with older age, longer duration of diabetes, history of $\mathrm{CV}$ disease and severe diabetic retinopathy (Table 1). Higher TN-C concentrations were also significantly associated with lower BMI, higher resting heart rate and higher serum concentrations of TNFR1 and ANGPTL2 (Table 1).

We found a weak negative correlation between baseline eGFR and TN-C $(\rho=-0.16, p<0.0001)$ and weak positive 
Table 2 Multivariate Cox analysis for the risk of all-cause death and MACE

\begin{tabular}{|c|c|c|c|c|}
\hline \multirow[t]{2}{*}{ Variable } & \multicolumn{2}{|l|}{ All-cause death } & \multicolumn{2}{|l|}{ MACE } \\
\hline & $\operatorname{HR}(95 \% \mathrm{CI})$ & $p$ value & $\operatorname{HR}(95 \% \mathrm{CI})$ & $p$ value \\
\hline Age (per year) & $1.06(1.05,1.07)$ & $<0.0001$ & $1.05(1.04,1.06)$ & $<0.0001$ \\
\hline Sex (reference: men) & $0.60(0.49,0.74)$ & $<0.0001$ & $0.62(0.51,0.75)$ & $<0.0001$ \\
\hline Active smoking & $1.40(1.02,1.92)$ & 0.0366 & $1.21(0.90,1.63)$ & 0.2123 \\
\hline Statin treatment & $1.00(0.82,1.21)$ & 0.9801 & $0.92(0.76,1.11)$ & 0.3834 \\
\hline Hypertension & $1.80(1.29,2.52)$ & 0.0006 & $1.59(1.17,2.16)$ & 0.0031 \\
\hline Insulin treatment & $1.44(1.16,1.78)$ & 0.0008 & $1.49(1.22,1.82)$ & $<0.0001$ \\
\hline eGFR (per $10 \mathrm{ml} \mathrm{min} \min ^{-1}[1.73 \mathrm{~m}]^{-2}$ ) & $0.92(0.87,0.97)$ & 0.0036 & $0.93(0.89,0.98)$ & 0.0106 \\
\hline History of MI and/or stroke & - & - & $1.37(1.10,1.69)$ & 0.0041 \\
\hline TN-C (per $1 \mathrm{SD})$ & $1.27(1.17,1.38)$ & $<0.0001$ & $1.23(1.13,1.34)$ & $<0.0001$ \\
\hline
\end{tabular}

correlations between baseline albumin/creatinine ratio (ACR) and TN-C $(\rho=0.10, p=0.0003)$ and between baseline CRP and TN-C $(\rho=0.19, p<0.001)$.

Compared with women, men had significantly higher mean TN-C concentrations $(78.3 \pm 45.6 \mathrm{ng} / \mathrm{ml}$ vs $72.3 \pm 40.1 \mathrm{ng} / \mathrm{ml}$; $p=0.0109$ ). However, since there was no significant statistical interaction of sex on the association of TN-C with outcomes, we presented results pooled by sex.

All-cause death and MACE Median follow-up duration was 89 months (57-130), corresponding to 9965 person-years. The overall mortality rate was $4.4 \%$ of total person-years $(95 \% \mathrm{CI}$ $4.0,4.8 ; n=442$ participants) and the MACE rate was $5.2 \%$ of total person-years (95\% CI 4.8, 5.7; $n=497)$. The major causes of death were CV diseases $(n=240 ; 54 \%)$, cancer $(n=74 ; 17 \%)$, infections $(n=39 ; 9 \%)$, renal causes $(n=4 ; 1 \%)$ and other causes $(n=85 ; 19 \%)$. Non-white ethnicity had no significant impact neither on death $(p=0.438)$ nor MACE $(p=0.699)$.

The Kaplan-Meier survival curve for all-cause death across TN-C tertiles is shown in Fig. 1a and for MACE in Fig. 1b. The comparable survival rates in the first two TN-C tertiles and the significantly lower survival rates in the third tertile are explained by the narrow distribution of serum TN-C levels in the tertiles 1 and 2 and a wide range of TN-C concentrations in the tertile 3. Baseline serum TN-C concentrations were higher in the group of individuals who died compared with survivors $(89.3 \pm 49.8 \mathrm{ng} / \mathrm{ml}$ vs $69.0 \pm 38.1 \mathrm{ng} / \mathrm{ml} ; p<$ 0.0001 ) and higher in individuals with incidental MACE compared with those without $(87.2 \pm 48.4 \mathrm{ng} / \mathrm{ml}$ vs $68.9 \pm$ $38.6 \mathrm{ng} / \mathrm{ml} ; p<0.0001)$.

After adjustment for predetermined risk factors, higher TN$\mathrm{C}$ concentrations were significantly associated with risk of allcause death (adjusted HR per 1 SD: 1.27 [95\% CI 1.17, 1.38]; $p<0.0001$ ) and MACE (adjusted HR per 1 SD: 1.23 [95\% CI 1.13, 1.34]; $p<0.0001$ ) (Table 2).

TN-C and discrimination Adding TN-C concentrations to these established prognostic factors (age, sex, active smoking, statin treatment, hypertension, insulin treatment, eGFR and history of $\mathrm{CV}$ disease) in type 2 diabetes modestly, but significantly improved the accuracy of the risk modelling for all-cause death (change in C-Statistics: 0.0111 [95\% CI 0.0107, 0.0114]; rIDI: $8.2 \%, p=0.0006$ ) and for MACE (change in C-Statistics: 0.0095 [95\% CI 0.0092, 0.0099]; rIDI: 6.7\%, $p=0.0014)$.

Sensitivity analyses After accounting for predetermined covariates, CRP plus TNFR1 and ANGPTL2 (adjusted HR per 1 SD of TN-C concentration: 1.15 [95\% CI 1.05, 1.27], $p=0.0025$ and 1.13 [95\% CI 1.03, 1.23], $p=0.0085$, for allcause death and MACE, respectively) or after accounting for predetermined covariates and NT-proBNP (adjusted HR per 1 SD: 1.16 [95\% CI 1.06-1.27], $p=0.0008$ and 1.13 [95\% CI $1.04,1.23], p=0.0041$, for all-cause death and MACE, respectively) in the survival model containing predetermined risk factors, the association findings were roughly unchanged for each outcome.

Additional adjustments for BMI, $\mathrm{HbA}_{1 \mathrm{c}}$, diabetes duration, LDL levels, SBP and the presence of diabetic retinopathy showed that the associations between serum TN-C concentrations and outcomes were robust and unchanged for each outcome (HR for 1 SD of TN-C: 1.25 [95\% CI 1.14, 1.36], $p<0.0001$ and 1.31 [95\% CI 1.19, 1.43], $p<0.0001$, for MACE and all-cause death, respectively).

\section{Discussion}

In this study we have shown that higher serum TN-C concentrations were significantly associated with an increased risk of all-cause death and MACE in individuals with type 2 diabetes after adjusting for predetermined risk factors. TN-C remained significantly associated with MACE and all-cause death, even after adjustment for inflammatory markers such as ANGPTL2 and TNFR1, which have been shown previously to be associated with these outcomes in type 2 diabetes [7, 8, 32-36]. This may suggest that the proinflammatory factor TN-C is 
independently associated with the development of diabetesrelated $\mathrm{CV}$ disease.

TN-C has been shown to be overexpressed in retinal endothelial cells cultured from diabetic human donors [25], as well as in retinal vascular basement membranes during long-term diabetes in an autopsy series [26]. Accordingly, we found that higher TN-C concentrations were associated cross-sectionally with more severe stages of diabetic retinopathy. Our results are also in accordance with a prior study, which shows that high serum concentrations of TN-C was weakly correlated with GFR [27]. In a population of 92 individuals with CKD (including $42 \%$ with type 2 diabetes), Liabeuf et al. reported that TN-C levels increased with the severity of CKD stage and could be used to predict MACE, CV death and all-cause death [27]. Of note, in this study the proportion of individuals with type 2 diabetes was not significantly different between groups with TN-C levels above/below the median [27]. In addition, serum TN-C concentrations have been reported to be significantly higher in individuals with diabetic nephropathy $(n=11)$ compared with healthy control participants $(n=176)$, even if no significant correlation between serum TN-C and creatinine clearance was found [37]. In our study, higher TN-C concentrations were not associated with diabetic nephropathy. In high-risk haemodialysed patients ( $38 \%$ with type 2 diabetes), serum $\mathrm{TN}-\mathrm{C}$ concentrations were found to predict all-cause- and CV-mortality [28]. In the latter study, high TN-C concentrations were associated with high blood glucose concentrations, and the authors attributed this relationship to the microvascular complications related to increased expression of TN-C (among other ECM components) that has been reported in the retina of individuals with diabetes [26]. Indeed, chronic inflammation related to diabetes seems to promote ECM remodelling and to induce the expression of 'diabetic-specific ECM proteins', including $\mathrm{TN}-\mathrm{C}$, which are not normally present in healthy retinal basement membranes [26].

In a prospective study performed in individuals after acute MI (38\% with type 2 diabetes), serum TN-C predicted MACE beyond the cardiac biomarker NT-proBNP [20]. Similar to our findings, in a cross-sectional study of individuals with or without CAD ( $41 \%$ with type 2 diabetes), elevated TN-C was associated with CAD history [23]. In a prospective study of individuals with dilated cardiomyopathy, myocardial expression of $\mathrm{TN}-\mathrm{C}$ was associated with type 2 diabetes [22]. In the latter study, high TN-C cardiac expression was associated with adverse left ventricular (LV) remodelling and overall mortality [22]. The authors proposed that diabetes, through TGF- $\beta$ signalling and inflammation, may promote cardiac ECM remodelling including TN-C expression [22], possibly contributing to the decline in LV function [22]. Interestingly, we showed that the prognostic value of TN-C persisted after adjustment for baseline history of $\mathrm{MI} /$ stroke and even after considering baseline NT-proBNP as a proxy of LV dysfunction (data not shown). Globally, a link between elevated TN-C concentrations and diabetes has been established in the kidney, retina and heart.

TN-C is considered as a damage-associated molecule pattern (DAMP) protein, which are associated with various chronic inflammatory diseases, including diabetes $[38,39]$. TN-C is an ECM protein and ECM remodelling occurs in both human and rodent models of diabetes and insulin resistance [40]. Chronic inflammation induced by obesity or hypoxia in the skeletal muscle, the liver and the adipose tissue is known to promote ECM remodelling. In these organs, increased ECM protein synthesis may contribute to insulin resistance through three hypothetical patways: (1) ECM may act as a physical barrier and prevent glucose and insulin diffusion; (2) ECM may impair vascular function and decrease vascular insulin delivery; (3) ECM may directly modulate insulin action [40]. In this model, inflammation-induced ECM remodelling promotes diabetes. Since TN-C overexpression is a key feature of inflammation-induced ECM remodelling, it seems plausible to assume that, in the SURDIAGENE cohort, TN-C could be involved, not only in the progression of CV-associated diabetes complications, but also in the pathogenesis of diabetes itself. The potential role of $\mathrm{TN}-\mathrm{C}$ as an aetiological factor in diabetes deserves further study.

In individuals with dysglycaemia, TN-C was found to be predictive of death only, but not MACE $[11,12]$. Interestingly, TN-C showed a significant predictive value in our cohort not only for all-cause death, but also for MACE, suggesting that $\mathrm{TN}-\mathrm{C}$ overexpression in individuals with type 2 diabetes might be linked to $\mathrm{CV}$ disease development and progression.

Our study has a number of potential limitations; for example, the French participants from the SURDIAGENE study were recruited in a prospective hospital-based monocentric cohort and this design may limit extrapolation of our findings.

The strengths of this study include an extended follow-up period and independent adjudication for outcomes. Samples were collected under standardised conditions and stored at $-80^{\circ} \mathrm{C}$ in a certified biobank, undergoing only one prior freeze-thaw cycle. To our knowledge, this is the first prospective study in type 2 diabetes reporting the association of serum $\mathrm{TN}-\mathrm{C}$ with risk of death or severe $\mathrm{CV}$ complications.

On the basis of the data collected in this prospective type 2 diabetes cohort, we conclude that increased serum TN-C concentration is independently associated with risk of allcause death and MACE, a finding facilitating improvement of risk prediction on top of traditional $\mathrm{CV}$ risk factors. Thus, TN-C could be a promising biomarker for improved risk stratification in individuals with type 2 diabetes. Further research 
will decipher whether TN-C is a potential therapeutic target in individuals with type 2 diabetes.

Acknowledgements All participants included and followed in the cohort study are warmly thanked for their kind participation in this research. Their general practitioners (GPs) are acknowledged for their help in collecting clinical information. E. Migault (INSERM CIC1402, Poitiers, France) and the staff of the Diabetes Department at Poitiers hospital are acknowledged for their help with data collection and monitoring. We thank A. Pavy, M.-C. Pasquier (Information Technology Department, CHU de Poitiers, Poitiers, France) and A. Neveu and J. Guignet (Medical Information Department, CHU de Poitiers, Poitiers, France). J. Arsham (CHU de Poitiers, Poitiers, France) carried out English language editing of the manuscript. A list of centres and staff involved in SURDIAGENE recruitment and adjudication is given as electronic supplementary material (ESM). Some of the data were presented as an abstract at the 30th European Meeting of the French Society of Cardiology (Journées européennes de la Société française de cardiologie, JESFC) meeting in 2020 .

Data availability The datasets generated during and/or analysed during the current study are available from the corresponding author on reasonable request.

Funding The SURDIAGENE cohort was supported by grants from the French Ministry of Health (PHRC-Poitiers 2004; PHRC-IR 2008), the Association Française des Diabétiques (Research Grant 2003) and the Groupement pour l'Etude des Maladies Métaboliques et Systémiques (GEMMS Poitiers, France). TN-C measurements were supported by a research grant from ELSAN, France (2016).

Authors' relationships and activities The authors declare that there are no relationships or activities that might bias, or be perceived to bias, their work.

Contribution statement BG conceived the work, obtained the grant for the measurement of TN-C and wrote the manuscript. NT-T conceived the work and wrote the manuscript. ET was involved with data analysis and interpretation and edited the manuscript. EG performed statistical analysis, contributed to drafting and revision of the manuscript. PS was involved in the study design, collected data, adjudicated clinical endpoints, and revised the manuscript. SB performed the assay for TN$\mathrm{C}$ and contributed to the drafting of the manuscript. SH conceived and constituted the SURDIAGENE cohort, collected data, was involved with data analysis and interpretation, and revised the manuscript. VR, DM, VJ, YP and PG adjudicated clinical endpoints, interpreted data and contributed to the drafting and revision of the manuscript. XP collected data and contributed to the drafting and revision of the manuscript. P-JS collected data, was involved with data analysis and interpretation and wrote the manuscript. All authors critically revised the article. BG and P-JS are the guarantors of this work and take full responsibility for the contents of the article.

\section{References}

1. Bachmann KN, Wang TJ (2018) Biomarkers of cardiovascular disease: contributions to risk prediction in individuals with diabetes. Diabetologia 61(5):987-995. https://doi.org/10.1007/s00125017-4442-9

2. Emerging Risk Factors Collaboration (2010) Diabetes mellitus, fasting blood glucose concentration, and risk of vascular disease: a collaborative meta-analysis of 102 prospective studies. Lancet
375(9733):2215-2222. https://doi.org/10.1016/S0140-6736(10) 60484-9

3. Bannister CA, Poole CD, Jenkins-Jones S et al (2014) External validation of the UKPDS risk engine in incident type 2 diabetes: a need for new type 2 diabetes-specific risk equations. Diabetes Care 37(2):537-545. https://doi.org/10.2337/dc13-1159

4. Hayes AJ, Leal J, Gray AM, Holman RR, Clarke PM (2013) UKPDS outcomes model 2: a new version of a model to simulate lifetime health outcomes of patients with type 2 diabetes mellitus using data from the 30 year United Kingdom Prospective Diabetes Study: UKPDS 82. Diabetologia 56(9):1925-1933. https://doi.org/ 10.1007/s00125-013-2940-y

5. Berezin AE, Kremzer AA (2013) Circulating osteopontin as a marker of early coronary vascular calcification in type two diabetes mellitus patients with known asymptomatic coronary artery disease. Atherosclerosis 229(2):475-481. https://doi.org/10.1016/ j.atherosclerosis.2013.06.003

6. Ozturk D, Celik O, Satilmis S et al (2015) Association between serum galectin-3 levels and coronary atherosclerosis and plaque burden/structure in patients with type 2 diabetes mellitus. Coron Artery Dis 26(5):396-401. https://doi.org/10.1097/MCA. 0000000000000252

7. Gellen B, Thorin-Trescases N, Sosner P et al (2016) ANGPTL2 is associated with an increased risk of cardiovascular events and death in diabetic patients. Diabetologia 59(11):2321-2330. https://doi. org/10.1007/s00125-016-4066-5

8. Saulnier PJ, Gand E, Ragot S et al (2014) Association of serum concentration of TNFR1 with all-cause mortality in patients with type 2 diabetes and chronic kidney disease: follow-up of the SURDIAGENE cohort. Diabetes Care 37(5):1425-1431. https:// doi.org/10.2337/dc13-2580

9. Price AH, Welsh P, Weir CJ et al (2014) N-terminal pro-brain natriuretic peptide and risk of cardiovascular events in older patients with type 2 diabetes: the Edinburgh type 2 diabetes study. Diabetologia 57(12):2505-2512. https://doi.org/10.1007/s00125014-3375-9

10. von Scholten BJ, Reinhard H, Hansen TW et al (2015) Additive prognostic value of plasma $\mathrm{N}$-terminal pro-brain natriuretic peptide and coronary artery calcification for cardiovascular events and mortality in asymptomatic patients with type 2 diabetes. Cardiovasc Diabetol 14:59. https://doi.org/10.1186/s12933-0150225-0

11. Gerstein HC, Pare G, McQueen MJ et al (2015) Identifying novel biomarkers for cardiovascular events or death in people with dysglycemia. Circulation 132(24):2297-2304. https://doi.org/10. 1161/CIRCULATIONAHA.115.015744

12. Gerstein HC, Pare G, McQueen MJ, Lee SF, Hess S (2017) Validation of the ORIGIN cardiovascular biomarker panel and the value of adding troponin I in dysglycemic people. J Clin Endocrinol Metab 102(7):2251-2257. https://doi.org/10.1210/jc. 2017-00273

13. Looker HC, Colombo M, Agakov F et al (2015) Protein biomarkers for the prediction of cardiovascular disease in type 2 diabetes. Diabetologia 58(6):1363-1371. https://doi.org/10.1007/s00125015-3535-6

14. Giblin SP, Midwood KS (2015) Tenascin-C: form versus function. Cell Adh Migr 9(1-2):48-82. https://doi.org/10.4161/19336918. 2014.987587

15. Midwood KS, Chiquet M, Tucker RP, Orend G (2016) Tenascin-C at a glance. J Cell Sci 129(23):4321-4327. https://doi.org/10.1242/ jcs. 190546

16. Chiquet-Ehrismann R, Chiquet M (2003) Tenascins: regulation and putative functions during pathological stress. J Pathol 200(4):488 499. https://doi.org/10.1002/path.1415 
17. Imanaka-Yoshida K (2012) Tenascin-C in cardiovascular tissue remodeling: from development to inflammation and repair. Circ $\mathrm{J}$ 76(11):2513-2520. https://doi.org/10.1253/circj.cj-12-1033

18. Midwood KS, Hussenet T, Langlois B, Orend G (2011) Advances in tenascin-C biology. Cell Mol Life Sci 68(19):3175-3199. https:// doi.org/10.1007/s00018-011-0783-6

19. Sato A, Aonuma K, Imanaka-Yoshida K et al (2006) Serum tenascin-C might be a novel predictor of left ventricular remodeling and prognosis after acute myocardial infarction. J Am Coll Cardiol 47(11):2319-2325. https://doi.org/10.1016/j.jacc.2006.03.033

20. Sato A, Hiroe M, Akiyama D et al (2012) Prognostic value of serum tenascin-C levels on long-term outcome after acute myocardial infarction. J Card Fail 18(6):480-486. https://doi.org/10.1016/j. cardfail.2012.02.009

21. Nozato T, Sato A, Hikita $\mathrm{H}$ et al (2015) Impact of serum tenascin-C on the aortic healing process during the chronic stage of type B acute aortic dissection. Int J Cardiol 191:97-99. https://doi.org/10. 1016/j.ijcard.2015.05.009

22. Yokokawa T, Sugano Y, Nakayama T et al (2016) Significance of myocardial tenascin- $\mathrm{C}$ expression in left ventricular remodelling and long-term outcome in patients with dilated cardiomyopathy. Eur J Heart Fail 18(4):375-385. https://doi.org/10.1002/ejhf.464

23. Gao W, Li J, Ni H et al (2019) Tenascin C: a potential biomarker for predicting the severity of coronary atherosclerosis. J Atheroscler Thromb 26(1):31-38. https://doi.org/10.5551/jat.42887

24. Kobayashi Y, Yoshida S, Zhou Y et al (2016) Tenascin-C promotes angiogenesis in fibrovascular membranes in eyes with proliferative diabetic retinopathy. Mol Vis 22:436-445

25. Castellon R, Caballero S, Hamdi HK et al (2002) Effects of tenascin-C on normal and diabetic retinal endothelial cells in culture. Invest Ophthalmol Vis Sci 43(8):2758-2766

26. To M, Goz A, Camenzind L et al (2013) Diabetes-induced morphological, biomechanical, and compositional changes in ocular basement membranes. Exp Eye Res 116:298-307. https://doi.org/10. 1016/j.exer.2013.09.011

27. Liabeuf S, Barreto DV, Kretschmer A et al (2011) High circulating levels of large splice variants of tenascin-C is associated with mortality and cardiovascular disease in chronic kidney disease patients. Atherosclerosis 215(1):116-124. https://doi.org/10.1016/ j.atherosclerosis.2010.11.038

28. Ulusoy S, Ozkan G, Mentese A et al (2015) A new predictor of mortality in hemodialysis patients; tenascin-C. Life Sci 141:54-60. https://doi.org/10.1016/j.lfs.2015.09.011

29. Hadjadj S, Fumeron F, Roussel R et al (2008) Prognostic value of the insertion/deletion polymorphism of the ACE gene in type 2 diabetic subjects: results from the non-insulin-dependent diabetes, hypertension, microalbuminuria or proteinuria, cardiovascular events, and Ramipril (DIABHYCAR), Diabete de type 2, Nephropathie et Genetique (DIAB2NEPHROGENE), and Survie, Diabete de type 2 et Genetique (SURDIAGENE) studies. Diabetes Care 31(9):1847-1852. https://doi.org/10.2337/dc07-2079
30. Levey AS, Stevens LA, Schmid CH et al (2009) A new equation to estimate glomerular filtration rate. Ann Intern Med 150(9):604 612. https://doi.org/10.7326/0003-4819-150-9-200905050-00006

31. Hicks KA, Tcheng JE, Bozkurt B et al (2015) 2014 ACC/AHA key data elements and definitions for cardiovascular endpoint events in clinical trials: a report of the American College of Cardiology/ American Heart Association Task Force on clinical data standards (Writing Committee to develop cardiovascular endpoints data standards). J Am Coll Cardiol 66(4):403-469. https://doi.org/10.1016/j. jacc.2014.12.018

32. Fernandez-Juarez G, Villacorta Perez J, Luno Fernandez JL et al (2017) High levels of circulating TNFR1 increase the risk of allcause mortality and progression of renal disease in type 2 diabetic nephropathy. Nephrology 22(5):354-360. https://doi.org/10.1111/ nep. 12781

33. Hata J, Mukai N, Nagata M et al (2016) Serum angiopoietin-like protein 2 is a novel risk factor for cardiovascular disease in the community: the Hisayama study. Arterioscler Thromb Vasc Biol 36(8):1686-1691. https://doi.org/10.1161/ATVBAHA.116.307291

34. Huang CL, Wu YW, Wu CC, Hwang JJ, Yang WS (2015) Serum angiopoietin-like protein 2 concentrations are independently associated with heart failure. PLoS One 10(9):e0138678. https://doi.org/ 10.1371/journal.pone. 0138678

35. Lee JE, Gohda T, Walker WH et al (2013) Risk of ESRD and all cause mortality in type 2 diabetes according to circulating levels of FGF-23 and TNFR1. PLoS One 8(3):e58007. https://doi.org/10. 1371/journal.pone.0058007

36. Neirynck N, Glorieux G, Schepers E, Verbeke F, Vanholder R (2015) Soluble tumor necrosis factor receptor 1 and 2 predict outcomes in advanced chronic kidney disease: a prospective cohort study. PLoS One 10(3):e0122073. https://doi.org/10.1371/journal. pone. 0122073

37. Horstrup JH, Gehrmann M, Schneider B et al (2002) Elevation of serum and urine levels of TIMP-1 and tenascin in patients with renal disease. Nephrol Dial Transplant 17(6):1005-1013. https:// doi.org/10.1093/ndt/17.6.1005

38. Piccinini AM, Midwood KS (2010) DAMPening inflammation by modulating TLR signalling. Mediat Inflamm 2010:672395. https:// doi.org/10.1155/2010/672395

39. Turner NA (2016) Inflammatory and fibrotic responses of cardiac fibroblasts to myocardial damage associated molecular patterns (DAMPs). J Mol Cell Cardiol 94:189-200. https://doi.org/10. 1016/j.yjmcc.2015.11.002

40. Williams AS, Kang L, Wasserman DH (2015) The extracellular matrix and insulin resistance. Trends Endocrinol Metab 26(7): 357-366. https://doi.org/10.1016/j.tem.2015.05.006

Publisher's note Springer Nature remains neutral with regard to jurisdictional claims in published maps and institutional affiliations. 